Revue d'histoire de l'Amérique française

REVUE D.HISTOIRE DE L'AMÉRIQUE FRANÇAISE

\title{
Le Père Frémiot à Thunder Bay, de 1848 à 1852
}

\section{Elizabeth Arthur}

Volume 25, numéro 2, septembre 1971

URI : https://id.erudit.org/iderudit/303065ar

DOI : https://doi.org/10.7202/303065ar

Aller au sommaire du numéro

Éditeur(s)

Institut d'histoire de l'Amérique française

ISSN

0035-2357 (imprimé)

1492-1383 (numérique)

Découvrir la revue

Citer cet article

Arthur, E. (1971). Le Père Frémiot à Thunder Bay, de 1848 à 1852. Revue

d'histoire de l'Amérique française, 25(2), 205-223.

https://doi.org/10.7202/303065ar d'utilisation que vous pouvez consulter en ligne.

https://apropos.erudit.org/fr/usagers/politique-dutilisation/ 


\title{
LE PĖRE FRÉMIOT À THUNDER BAY, DE 1848 À 1852
}

\author{
Elizabeth Arthur \\ Lakehead University \\ Thunder Bay
}

C'est vers la fin des années 1840 que les missions des Jésuites, dans la région que l'on nomme aujourd'hui Thunder Bay, eurent leur début. A l'origine, pendant une courte période, un jeune prêtre français fut intimement lié à cette nouvelle mission. Le Père Nicolas Frémiot, né à Bellefontaine, dans le Département des Vosges, en 1818, avait été promu sous-diacre en septembre 1846 , et prêtre une année plus tard. Aussitôt ordonné, il quittait l'Europe pour les missions en terre canadienne. Il passa un hiver dans la région de Montréal avant de continuer sa route vers le Haut-Canada. Il se dirigea ensuite vers Pigeon River, à l'extrémité ouest du lac Supérieur, près de la frontière américaine. De ce port d'attache, puis, de la mission de Fort William, il se mit au service des mineurs canadiens qui travaillaient dans des centres isolés, sur le lac Supérieur et sur l'île Royale, desservant également, jusqu'aux limites du lac Nipigon, les comptoirs de la Compagnie de la Baie d'Hudson, où se faisaient les échanges de fourrures entre Indiens et commerçants.

D'après son propre récit, le Père Frémiot abandonna derrière lui, à Sandwich (la seule paroisse organisée de l'Ouest), la région du Haut-Canada où aucun travail missionnaire n'avait été fait jusqu'alors, et se dirigea avec deux compagnons au SaultSainte-Marie. ${ }^{1}$ Le 15 juillet 1848, ils se mirent en route le long de la côte du lac Supérieur, s'arrêtèrent à la Pointe (Wisconsin) où ils visitèrent le Père Frédéric Baraga, linguiste slovène, qui y habitait et qui sera plus tard le premier évêque du Michigan supérieur. ${ }^{2}$ Une lettre du Père Frémiot, datée du 24 juillet, décrit leur arrivée à destination, la rivière aux Tourtres. ${ }^{3}$

${ }^{1}$ Archives de la Société de Jésus du Canada Français (A.S.J.C.F.), Lettres de Nouvelles Missions du Canada, 1845-1852, I: 372-78, P. Frémiot au Provincial à Paris, le 24 juin 1848.

2 Sister Mary Aquinas Norton, Catholic Missionary Activities in the Northwest, 1818-1864 (Washington, 1930), 72-3.

3 A.S.J.C.F., Nouvelles Missions, I: 380-81, P. Frémiot à un père Jésuite, le 24 juillet 1848.

[205]

RHAF, vol. 25, no 2 (septembre 1971) 
Le mercredi à midi nous débarquions a Princess Bay ${ }^{4}$ avec une compagnie de mineurs canadiens conduit là par $\mathrm{M}$. Robertson de Montréal. On y exploite depuis deux ans une mine d'argent et de cuivre. Ce messieur, quoique protestant, fut aux petits soins pour les trois Jésuites...

Vers midi nous entrons dans cet autre Jourdain qui arrose notre terre promise. Mais ici pas de Jebusien à combattre, personne pour nous en disputer la paisible possession. En effet, nous mettons pied-à-terre et que voyons-nous ? Là et là quelques maisons inachevées, ailleurs les perches encore debout d'une loge devenue déserte puis, sur un plateau central, au bord de la rivière, une église en bois à laquelle il ne manque jadis que la couverture, mais qui maintenant tombe pièce à pièce; un peu plus loin une maison ou, si vous aimez mieux, une cabine qui se compose d'un seul appartement qui laisse voir le ciel à travers les fentes de la toîture. C'est la maison qui nous est destinée. Un missionnaire, M. Pirse [sic] la fit bâtir ainsi que l'Eglise il y a cinq ans; puis effrayé des difficultés qu'offrait alors la mission il l'abandonne pour aller au Sault Ste. Marie; il est aujourd'hui à l'Arbre Croche, missioñ d'Ottâwas sữ le lac Míchigan.

Par cette lettre, on aperçoit un bon nombre des caractéristiques de la correspondance du Père Frémiot faite dans les années qui suivirent, à savoir: une description graphique et détaillée de ce qu'il voyait, mêlant aux tableaux les plus désolés un sens de l'humour qui enjolivait même ce qu'il y avait de plus sombre. Cette correspondance ne manque pas d'exprimer la reconnaissance du Père au sujet des faveurs qu'il recevait des dirigeants de mines, même protestants, non plus que sa conviction qu'il n'y avait aucune autorité qui puisse inquiéter les Jésuites dans leur terre promise - exprimant parfois comme évidentes les suppositions des ouï-dire. Que le Père Frémiot ait pris le nom "Prince's Bay" pour celui de "Princess Bay", dans une langue qui lui était étrangère, n'entache aucunement la validité de son récit, d'autant plus qu'il employa ce nom correctement dans les lettres qui suivirent. ${ }^{5}$ Plus graves de conséquences furent ses interprétations au sujet des raisons d'agir du Père Francis Pierz, qu'il ne corrigera pas, probablement parce que toute l'information, si mince fût-elle qu'il ait jamais eue sur la personnalité de cet homme, lui était déjà connue avant 1848.

${ }^{4} \mathrm{Ce}$ centre minier, à quelques milles au nord-ouest de l'embouchure de la rivière Pigeon, fut concédé au colonel John Prince de Sandwich (plus tard de Sault Sainte-Marie) en 1846. C'est sous ce nom qu'on le connaissait dans la région.

5 A.S.J.C.F., Nouvelles Missions, I: 409, P. Frémiot à un Jésuite, le 8 décembre 1848; Ibid., 424, P. Frémiot au Supérieur à Montréal, le 16 avril 1849 . 
L'histoire véridique de l'œuvre du Père Pierz dans la région de Thunder Bay, toutefois, laisse une impression bien différente de celle qu'on peut tirer du récit plus ou moins soigné du Père Frémiot. Dès 1838, le Père Pierz avait établi une mission à GrandPortage, sur la frontière américaine, à partir de laquelle il visita en plusieurs occasions, les comptoirs de fourrures, à Fort William. On lui avait donné l'ordre d'aller à Arbre Croche, mais il ne cessa de considérer les Indiens ojibways comme étant supérieurs aux Ottawas en talent et en zèle, et de demander la permission de retourner auprès d'eux. En 1842, on lui avait permis d'établir une mission à Pigeon River, dont le Père Frémiot décrira les ruines six ans plus tard. Il avait fondé là une école pour quarante-cinq enfants indiens quand il fut rappelé à Arbre Croche. Le Père Pierz fit sa dernière visite à Pigeon River en 1847, et en route vers le lac Michigan, rencontra à La Pointe un supérieur des Jésuites canadiens avec qui il s'entendit pour faire poursuivre son œuvre par d'autres personnes. ${ }^{6}$

Immédiatement après son arrivée, le Père Frémiot fut assez sage pour ne pas porter de jugement sur la situation et assez discret pour ne glisser dans sa correspondance que peu de remarques critiques sur ses associés. C'était cependant un homme aux opinions fermes et à la langue colorée. Ses lettres n'étaient ni froidement analytiques ni objectives. Presque chaque ligne laisse paraître un jugement de valeur. Ce ton subjectif ajoute à ses lettres beaucoup d'intérêt et de charme, mais il comporte par là même un certain danger. Il n'est pas toujours possible de vérifier les événements qu'il décrit. Faute de biographes sérieux pour rétablir leur réputation, les chefs indiens et les petits commerçants durent, en bien des circonstances, laisser le lecteur se fier au seul témoignage de la relation du Père Frémiot. Néanmoins, on peut voir qu'une longue fréquentation de certaines personnes, ainsi que la mise en présence des faits portant sur une période assez longue, ont conduit le Père Frémiot à réévaluer ses impressions et à rééquilibrer son jugement, dans un effort de volonté qui lui fit dépasser la nature émotionnelle d'une réaction première, vouée aux variations de ses propres humeurs.

En conséquence, ce qu'il écrit sur les deux hommes qui l'accompagnaient en 1848 et qui allaient travailler près de lui pendant les quatre années suivantes, mérite quelques vérifications. Il décrit le Frère Frédéric de Pooter plutôt affectueusement comme un "fortissimus Belga", manquant quelque peu de prudence et d'esprit d'obéissance, et peu expérimenté dans le maniement de la

6 Norton, op. cit., 66-71. 
hache. ${ }^{7}$ Le dernier aspect de ce jugement semble faire allusion à une coupure que le valeureux Frère s'était faite avant que les réparations de la mission n'aient même été commencées, ${ }^{8}$ et il se pourrait bien que les commentaires au sujet de ses défauts de tempérament fussent aussi rattachés au même incident. Quoi qu'il en soit, les deux hommes continuèrent à travailler assez amicalement ensemble à Thunder Bay et à Sault-Sainte-Marie. De Pooter, dont l'expérience au Canada avait également commencé en 1847, fut envoyé à Fordham en 1854 pour y rester jusqu'à sa mort, quelques années plus tard..$^{9}$ Naturellement, sa notice nécrologique ne contient aucune des mentions, sous-entendues dans le récit du Père Frémiot, à son inadaptation au travail dans le nord. Mais une comparaison de deux notices est significative à ce sujet: d'une part, l'une d'elles traite de façon succincte les années de services du Frère de Pooter, tandis que l'autre s'étend avec complaisance et détails sur la faculté d'adaptation du successeur de ce dernier, à Thunder Bay, le Frère Dominique Leischner. ${ }^{10}$

Lê Pèrê Choñé était, en âge et expérience, l'ainé des Jésuites qui arrivèrent au Canada en 1848. Il avait travaillé à l'île Manitouline pendant quatre ans et avait déjà maîtrisé avec succès les langues indiennes que le Père Frémiot commençait juste à étudier. Presque toutes les premières mentions écrites du Père Frémiot, au sujet du Père Choné, parlent de son aptitude pour les langues et de son intérêt à la musique. Le déplorant plutôt, le Père Frémiot fait observer que, pendant des voyages fatigants, les Père Choné et Baraga discutaient de neuf heures du soir à deux heures du matin de la complexité d'une traduction de concepts théologiques en ojibway. ${ }^{11}$ Quelques mois plus tard, on peut lire la description très suggestive de la messe de "Tous les Saints" à Pigeon River: "L'honneur de célébrer me fut dévolu, le $\mathrm{P}$. Choné était nécessaire à l'orchestre. Accompagné de quelques sauvages, il chanta en leur langue le Gloria in excelsis et le Credo." ${ }^{2}$ Quant le Père Frémiot eut maîtrisé la langue indienne, il fut tout à fait prêt à concéder que son supérieur, le Père Choné,

${ }^{7}$ A.S.J.C.F., Nouvelles Missions, I: 397, P. Frémiot au Provincial à Paris, le 11 août 1848.

8 Ibid., II : 178, P. Frémiot à un Jésuite en France, le 3 décembre 1851.

9 Woodstock Letters (Woodstock College, 1872-), XX (1891): 289, Notice nécrologique du Père de Pooter.

10 Ibid., XXXVII (1908) : 398-9, Notice nécrologique du Père Leischner.

11 A.S.J.C.F., Nouvelles Missions, I : 379, P. Frémiot à un père Jésuite le 24 juillet 1848 .

12 Ibid., I: 416, R. P. Frémiot à un père Jésuite, le 8 décembre 1848 . 
était plus doué que lui pour la traduction et qu'il le dépassait dans l'enseignement aux enfants indiens. "C'est le Père Choné qui en sa qualité de musicien adapte le chant Romain aux traductions que nos sauvages ont dans leurs livres; c'est lui aussi qui leur fait la classe tous les jours." ${ }^{13}$ Visitant l'île Manitouline pour la première fois, le Père Frémiot rapporte les gentilles remarques qu'il entendit au sujet de son associé: "Notre Père qui parle fort". ${ }^{14}$

En quatre ans de proche collaboration, le Père Frémiot fait à peine une allusion au tempérament de son supérieur; les compliments, quand il en fait, sont au sujet d'un point de peu d'importance et quelquefois superficiel. Le rapport officiel de la mission de Thunder Bay, qui parut bien tard, pourrait expliquer cette discrétion : ${ }^{15}$
Le supérieur [Choné] a d'excellentes qualités, volonté forte, cœur excellent, courage, ponctualité parfaite. Son caractère rigide pour lui-même et trop pour les autres, l'éprouve lui- même et surtout les autres. Son principe est toujours le zèle de l'Ordre. Il ne sait pas assez fléchir, consoler, encourager ... Il veut trop la pauvreté et l'économie, et même la mortifi- cation dans la vie commune. La fermeté, une certaine sévérité est nécessaire avec les sauvages mais il y [?] en apparence d'irritation nerveuse qui empêcha l'onction de sa parole. C'est le règne de la crainte plus que de l'amour.

Le contraste entre les personnalités du Père Choné et du Père Frémiot était très marqué. Leur apparente amitié peut s'expliquer du fait qu'ils ne restèrent jamais longtemps ensemble pour travailler. La preuve: cette amitié ne sera pas sauvegardée par les successeurs du Père Frémiot. ${ }^{16}$ Durant leur premier été à Pigeon River, le Père Choné s'absenta plusieurs semaines pour la Pointe où les Indiens étaient rassemblés afin de recevoir les annuités du gouvernement américain. Au cours de cette absence, le Père Frémiot qui consacrait son temps à l'étude d'une grammaire indienne, se trouva plongé dans une excessive solitude ${ }^{17}$ que même le retour du Père Choné ne soulagea que partiellement.

13 Ibid., I : 417, P. Frémiot à un Jésuite, le 8 décembre 1848; II : 118, P. Frémiot au Supérieur à Paris, le 15 septembre 1851.

14 Ibid., II : 30, P. Frémiot au Provincial à Paris, le 27 septembre 1850.

15 A.S.J.C.F., Missions Indiennes, Rapport de la visite des missions:

Ile Manitouline, Sault-Ste-Marie, Fort William. Jugement sur chacun des missionnaires. P. Nicolas Point à R. P. Supérieur, le 15 septembre 1859.

16 Ibid., Jugement sur P. Ferrart.

le 7 août 1848 . 
Le printemps suivant, l'un d'eux alla passer dix jours à Fort William, tandis qu'à son retour, l'autre partait pour une visite de tous les camps miniers, depuis la région de Prince's Bay jusqu'à ceux de l'île Royale. La décision prise plus tard en cours d'année de transférer le centre missionnaire à Fort William signifiait encore une autre séparation, car l'un des Pères devait superviser les constructions, tandis que l'autre resterait à Pigeon River tant que les édifices du nouvel emplacement ne seraient pas terminés.

Une légende a voulu que le mérite de la construction de la mission revienne au Père Choné. L’origine de cette légende pourrait bien être attribuée à deux ministres méthodistes qui visitèrent la mission de Fort William et publièrent sa description en 1850. Le révérend Peter Jacobs, un pasteur indien, fit son apparition en 1852, pendant que le Père Frémiot était à Nipigon. Il causa avec le Père Choné qu'il décrit comme s'exprimant assez mal en anglais, mais parlant "tolerably good Indian". ${ }^{18}$ Il confie à son journal:

He was busy raising the frame of a chapel and will likely, in process of time, make a good mission of this; though new newly established, it is getting on wonderfully and the Indians are living in their wigwams.

Deux ans plus tard, le révérend John Ryerson décrivait ces bâtiments avec beaucoup plus de détails. Il avait éprouvé alors de grandes difficultés à communiquer avec le Père qu'il nomme Monsieur "Shuny". Il s'en est suivi un récit trompeur. ${ }^{19}$

The building... was accomplished principally by Mr. S's own hands, and during the same time he performed his ordinary work as priest, besides assisting in the day school, visiting it twice every day, catechizing and instructing the children.

Quelle qu'ait été la concordance de ce tableau avec ce qui existera plus tard, les lettres du Père Frémiot disent clairement qu'il n'y eut pas d'école à Fort William avant 1851, et que c'est lui qui était resté là à l'automne de 1849 pour superviser les débuts de la construction. Il décrit ainsi la construction de la première cheminée, performance qui aurait confondu, il en était sûr, les architectes et les maçons de l'Europe, s'il leur avait été possible de voir ce travail: ${ }^{20}$

18 Rev. Peter Jacobs, Journal (Toronto, 1854) 19, 27 mai 1852.

19 Rev. John Ryerson, Hudson Bay - a missionary tour (Toronto, 1855), 18, entrée du 24 juin 1854, dans une lettre du 3 juillet 1854 au Rev. Enoch Wood, président de la "Wesleyan Conference".

20 A.S.J.C.F., Nouvelles Missions, II: 3-4, P. Frémiot au Provincial à Paris, janvier 1850. 
On commence par planter en terre quatre perches de la hauteur de la cheminée; car on a eu soin de laisser le toit vide assez considérable, qui ne sera rempli qu'après la cheminée faite, ces quatres perches souliées ensemble par des barres transversales distantes d'un demi-pied l'une de l'autre, ce qui forme une espèce d'échelle pyramidale. Tel est le squelette de la cheminée. Pour la garnir on enferme de la glaise mêlée de sable, dans un petit rouleau de foin, qu'on presse, qu'on manie jusqu'à ce que la glaise le compénètre et ressorte par le bas. Un second vient s'accoler au premier, plus un troisième au second, et ainsi toute la cheminée s'élève, unie, solide et compacte.

Durant les deux premières années de son séjour à Thunder Bay, le Père Frémiot posa des fondations de plus d'une façon. Il maîtrisait suffisamment les langues indiennes pour entreprendre ses longs voyages missionnaires de 1851 et de 1852 . Il s'était habitué aux conditions d'une vie dure dans un climat assez hostile. On l'a considéré comme l'un des premiers à faire des visites régulières dans les camps miniers, où, par exemple, il fut bouleversé de constater que, jusqu'alors, les hommes n'avaient pas joui du privilège des fêtes religieuses chômées. ${ }^{21}$ Il avait participé à la mise sur pied d'un centre missionnaire permanent à Fort William. Convaincu que le tempérament nomade des Indiens était le principal obstacle au travail des Jésuites à Thunder Bay, il s'efforça de les persuader de s'établir dans les environs de la mission. John McKenzie, gérant de vente de la Compagnie de la Baie d'Hudson à Thunder Bay, reconnaissait les mérites de la nouvelle mission, réclamée avec instance depuis $1841 . .^{22}$ Il était en très bons termes avec les Pères qui étaient là, ${ }^{23}$ et il rapporte dans ses comptes rendus certains gestes pratiques qui l'influencèrent dans sa gestion des affaires. Le 15 août 1850, il écrit: "Being a holiday among the Catholics, the men at the Post attended Mass, but in the afternoon they were sent to work at the Hay." ${ }^{24}$ Plusieurs mois plus tard, John McKenzie note: "All the People who are Catholics were sent up today to chop Cordwood for the church. The others were employed at various

21 Ibid., I: 409, P. Frémiot à un père Jésuite, le 8 décembre 1848.

22 Sir George Simpson, Narrative of a Journey Round the World during the years 1841 and 1842 (London, 1847) I : 35, 28 mai 1841.

23 A.S.J.C.F., Nouvelles Missions, I: 443, P. Frémiot au Supérieur à New York, le 18 octobre 1849; II: 5, P. Frémiot au Provincial à Paris, janvier 1850; II: 148, P. Frémiot à un Jésuite scolastique, le 18 octobre 1851.

24 Public Archives of Canada (P.A.C.), Microfilm of Hudson's Bay Company Records, B-231-a-20, Fort William Post Journal, 1849-51 (August 1850). La Compagnie de la Baie d'Hudson nous a gracieusement accordé la permission de citer ses archives. 
duties." ${ }^{25}$ On trouve une image beaucoup plus détaillée et colorée de cette étape missionnaire dans la description que fait le Père Frémiot de la première procession de la Fête-Dieu qui eut lieu à Thunder Bay, en 1850: ${ }^{26}$

La procession fut renvoyée au dimanche suivant à cause du mauvais temps. Ce jour-là deux reposoirs bien modestes furent dressés. Deux beaux sapins décoraient à l'intérieur la maison de la prière, d'autres ombrageaient la façade et bordaient la place, qui est à l'entrée ainsi que les avenues qui conduisaient aux reposoirs. Comme presque aucun des assistants ne savait ce que c'était qu'une procession ce ne fut pas sans peine que je parvins à organiser une marche un peu régulière, mais par la même raison, quelque modeste que fut cette fête religieuse au milieu des forêts, c'était un spectacle magnifique pour les sauvages, ils en furent tous dans l'admiration.

$\mathrm{Au}$ milieu de l'été 1850 , la première partie de l'œuvre du Père Frémiot prenait fin. Il quittait Fort William, le 19 juillet, à bord de la goélette de la Compagnie de la Baie d'Hudson, le White $F i s h$, poür un voyage de six jours au Sault. Ensuite, après avoir visité l'île Manitouline, il remonta la rive sud, route qu'il avait suivie deux ans plus tôt, et resta quelque temps sur l'île Royale avant de retourner à Fort William à la fin de septembre. ${ }^{2 \tau} \mathrm{A}$ partir de ce moment, ses lettres ne traitent que de facon intermittente de la mission de Fort William. Il devenait de plus en plus préoccupé du rôle de missionnaire dans toute la région de Thunder Bay. C'est dans cette perspective qu'il considéra les conditions du bien-être général des Indiens, et réexamina ses premières impressions sur les questions économiques et sociales, de même que les plans d'action qu'elles engendraient.

Il fut évident dès le début que le Père Frémiot considérait d'une façon quelque peu différente de celle du Père Choné la possible rivalité entre les missionnaires d'autres croyances. On pourrait attribuer cette divergence de vues aux différences de tempérament et d'expérience. Pendant les années vécues sur l'île Manitouline, le Père Choné avait eu des contacts malheureux avec les missionnaires anglicans du pays, ${ }^{28}$ aussi bien qu'avec les méthodistes d'Owen Sound qu'il décrivait habituellement

25 Ibid., (January 3, 1851).

26 A.S.J.C.F., Nouvelles Missions, II: 26, P. Frémiot à un prêtre Jésuite, le 21 juin 1850.

27 Ibid., II : 30, P. Frémiot au Provincial à Paris, le 27 septembre 1850.

28 Ibid., I: 110, P. Choné au Supérieur en France, le 3 septembre 1844; $\mathrm{I}: 399, \mathrm{P}$. Choné à un père Jésuite, le 14 septembre 1848. 
comme "séduisants". ${ }^{29}$ Au cours de ses premières années à Thunder Bay, il resta convaincu, tout au moins, que la grande menace à l'entreprise jésuitique était due aux activités de ces rivaux. Bien que, d'une part, grâce à son sens politique, le Père Frémiot perçut ce danger hypothétique, il se rendait compte, néanmoins, de la faiblesse du protestantisme à Thunder Bay. Il usa de beaucoup de diplomatie dans ses contacts avec les mineurs, protestants et catholiques, ainsi qu'avec les administrateurs de mines, pour la plupart protestants. Avec un rire un peu jaune, il décrit le genre de rassemblement liturgique qui l'attendait à quelquesunes des mines; il compta ainsi trois mineurs protestants, quatre Indiens et deux femmes indiennes (religion non spécifiée), ne faisant allusion à personne d'autre. ${ }^{30} \mathrm{~A}$ plusieurs reprises, il a louangé les surintendants généraux des mines pour leur coopération et leur gentillesse avec lui. ${ }^{31}$ Dans une lettre adressée au Provincial de Paris, il notait sans plus de commentaires une cérémonie à la mine Cliff qui put sembler peu commune à celui qui reçut la lettre: "Il y a là un beau temple protestant où $\mathrm{Mgr}$ du Détroit dit la messe et prêcha ce printemps-là lors de sa visite de confirmation à l'Anse." ${ }_{22}$ Queelles qu'aient été les vues du Père Frémiot sur l'œcuménisme forcé, près de la frontière, il était à même de se rendre compte que les mines de la rive nord du lac Supérieur étaient en train de disparaître rapidement. Une courte période de l'histoire de la vie économique de la région venait de se terminer vers 1850 , et toutes les menaces que représentait l'immigration protestante à cause des mines, de même que la puissance politique qu'elles impliquaient, étaient disparues à ce moment-là.

Le Père Frémiot semble avoir trouvé amusants plutôt que sinistres ses contacts avec les Indiens qui se réclamaient d'une secte protestante. Du chef illinois, il écrit: ${ }^{33}$

Il y a un certain nombre d'années qu'un ministre baptiste de Sault le plongea dans la rivière avec quelques membres de sa famille. C'est là tout ce qu'on lui a appris de la religion, car il sait absolument rien.

29 Ibid., I: 267, P. Choné aux étudiants du Collège de Brugelette, le 7 août 1847 . 1851.

30 Ibid., II: 156, P. Frémiot à un scolastique Jésuite, le 18 octobre 1850 .

31 Ibid., II: 157; II: 25, P. Frémiot à un prêtre Jésuite, le 21 juin

32 Ibid., II: 38, P. Frémiot au Provincial à Paris, le 27 septembre 1850. 1849.

33 Ibid., I: 450, P. Frémiot au Supérieur à New York, le 18 octobre 
Deux ou trois soi-disant méthodistes parmi les Indiens de Fort William ont pu se montrer gênants en s'opposant à la construction de l'église. Dans un rêve, l'un d'eux avait eu la révélation que si elle s'établissait à proximité de la mission, la tribu serait gravement menacée en temps d'épidémie. Une semblable épidémie a même, de fait, causé plusieurs morts à l'automne 1849, et le Père Frémiot était parfaitement conscient que les Indiens ne prenaient pas les rêves à la légère. ${ }^{34} \mathrm{Au}$ dire du Père, ce rêve, quelle que fût son horreur, n'avait pas empêché les méthodistes d'accepter du travail sur les chantiers de construction. C'est à croire que seules la chapelle et les maisons terminées pouvaient avoir l'effet nocif qu'ils craignaient.

Ce que l'on sait maintenant, et le Père Frémiot s'en est rendu compte au cours de ses voyages, c'est qu'aucune mission protestante n'avait été fondée avant celle-ci, dans tout le district de Thunder Bay. Ni les Anglicans, ni les Baptistes ne s'étaient avancés au-delà du Sault-Sainte-Marie. Le révérend Thomas Hulburt, méthodiste, s'était rendu, en 1838, jusqu'au Pic, où se trouvait un comptoir de la Compagnie de la Baie d'Hudson. $\mathrm{Au}$ cours des quelques années qui suivirent, il visita à l'occasion Fort William, mais une décennie plus tard, les vestiges de son ministère n'auront plus rien de très significatif. A l'époque où le Père Frémiot était à Thunder Bay, le révérend Peter Jacobs se trouvait dans la région de Rainy River, mais il n'y a aucune preuve que les deux hommes se soient jamais rencontrés. Le témoignage le plus impressionnant en ce qui concerne les forces et les faiblesses relatives de l'apostolat catholique et méthodiste dans le nord nous est transmis par le révérend John Ryerson. Après avoir visité la mission de Fort William, en avoir observé les bâtiments, le fonctionnement de l'école, et pardessus tout, le logement austère et le dévouement désintéressé du Père Choné, il écrit: 35

I remarked to by brethren that such laborious and selfsacrificing zeal was worthy of a better cause than the spreading of Popery, and that it was humiliating to ourselves, contrasted with the exertion and success of some of our own missionaries, who seem to measure out their work, both with regard to kind and extend, with as much care and exactness as a Jew would measure silk velvet. I recollect one missionary, if not more than one, who objected to teaching a day-school on the ground that it was not quite canonical, and was beneath the dignity of the ministerial office; that

34 Ibid., II: 2-3, P. Frémiot au Provincial à Paris, janvier 1850. 35 Ryerson, op. cit., 19. 
he was called to be a minister not a school teacher. One thing is certain, that the Roman Catholic missionaries throughout these extensive regions, in zeal, in labour, in self-denial, and in success in their work, are much, very much, before us, and unless we bestir ourselves with very much more united, earnest, and persevering exertion than what we yet have, this whole country will be overspread and hedged in with the briars, thorns and hedges of Popery.

Témoin, sur l'île Royale, de conditions de vie austères, le Père Frémiot avait réagi de façon très différente: ${ }^{36}$

Mais, qu'on est ici logé à l'étroit, au milieu de ces vastes déserts ! Le docteur MacKulagh [sic] presbyterien, intendant de la mine, n'a pour chambre à coucher, pour cabinet et pour salon qu'un misérable coin de magasin où il est comme perdu au milieu des tonneaux et des provisions. Un lit, une petite table et quelques livres, un seul banc en guise de chaise, c'est là tout son ameublement. Peut-on plaindre la pauvreté de ceux qui se dévouent à la conquête des âmes tandis que les amateurs de l'or ne craignent pas, pour l'espoir incertain d'un vil métal, de s'astreindre à si dures privations ?

L'absence d'allusions sectaires dans les lettres du Père Frémiot peut sans doute s'expliquer en partie par le fait qu'il était étranger à la scène politique canadienne. Tandis que le Père Choné conservait des souvenirs amers de ses luttes sur l'île Manitouline, et que Ryerson restait également déçu de ses controverses politiques et religieuses dans la province soi-disant unie du Canada, le Père Frémiot, lui, était demeuré à l'écart des conflits d'opinions qui avaient cours loin de Thunder Bay. Le Canada français, pour lui, n'avait rien de politique. Son séjour dans la région du Saint-Laurent avait été bref, et le nom de Mgr Bourget n'avait rien de plus explosif, politiquement parlant, que le soin des orphelins irlandais à Montréal. ${ }^{37}$ Les quartiers généraux de son Ordre en Amérique se trouvaient à New York, ce qui ne signifiait ni penchant en faveur des Etats-Unis, ni préjugé contre eux. Qui plus est, son départ de la France à l'automne 1847 l'avait délivré, pour une bonne part, de la crise de conscience que la révolution de 1848 engendra chez les catholiques européens. Son propre passé, aussi bien que l'isolement géographique dans lequel il vivait, l'avaient ainsi amené à considérer les problèmes de la vie missionnaire à la lumière des facteurs de l'environnement et de la culture.

36 A.S.J.C.F., Nouvelles Missions, I: 431, P. Frémiot au Supérieur à Montréal, le 16 avril 1849. 1847.

37 Ibid., I: 319-28, P. Frémiot au Provincial à Paris, le 27 décembre 
La description d'un voyage fait au printemps de 1850 met clairement à jour des difficultés de cet ordre. Le 24 avril, trois hommes venant de la part du chef indien de Pigeon River arrivèrent à Fort William, rapportant que plusieurs de ses enfants étaient morts, et implorant le Père Choné de venir administrer les derniers sacrements à sa petite fille qui était gravement malade. ${ }^{38}$

Le $\mathrm{P}$. Choné est en retraite; il m'envoie à sa place. La difficulté est de trouver un homme pour porter ma chapelle; presque tous les sauvages sont à la chasse, et c'est la saison la plus mauvaise et la plus dangereuse pour voyager sur le lac dont la glace commence à se fondre et à se briser. Encore 8 ou 10 jours, et la navigation sera facile, mais la mort sera-t-elle assez complaisante pour attendre jusque là avant de frapper la malade qui réclame le secours de mon ministère ?

Suit la narration d'un voyage invraisemblable au terme duquel le Père Frémiot trouve la maison du chef abandonnée. Cette désertion, il l'avait appris, signifiait que l'enfant était déjà mort et que les parents, suivant leur coutume, étaient partis. Il se mit à vérifier le cimetière à la recherche d'une tombe frâ̂chement recouverte, mais ses recherches ne menèrent à rien, puisque tous les alentours étaient recouverts d'écorces de bouleau. Néanmoins, il récita le $D e$ profondis. Il se rendit compte alors que toute la colonie avait évacué, et qu'à cause d'une pénurie de nourriture, les Indiens avaient déménagé à Grand-Portage. Marchant à leur trousse, il continua de chercher le chef, profitant du voyage pour entendre les confessions. "Comme je n'avais point de grille, écrit-il, je conçus l'idée d'improviser une avec deux raquettes fichées en terre, et je n'eus qu'à m'applaudir de l'invention." Finalement, il découvrit l'enfant âgé de deux ans, et bien portant. Puis, il revint lentement vers Fort William. Si la mort n'attend pas, une tournée de confessions en pareilles circonstances aurait bien pu, elle, attendre.

Un an et demi plus tard, dans une longue lettre à un Père jésuite français, non nommé, le Père Frémiot essayait d'analyser les obstacles que les missionnaires de Thunder Bay ont à surmonter. ${ }^{39}$ Il classa ces difficultés comme il les vit, en ordre décroissant d'importance: la vie nomade des Indiens, la pratique de la polygamie, l'attachement des Indiens à des traditions 1850.

${ }^{38}$ Ibid., II : 13-20, P. Frémiot aux Scolastiques à Laval, le 11 mai

39 Ibid., II : 168-178, P. Frémiot à un père Jésuite en France, le 3 décembre 1851 . 
bien particulières, et surtout, le cérémonial de la danse (qu'il croyait être en train de mourir, sauf dans ce centre de perversité qu'était Rainy River), et enfin, la vente de l'alcool aux Indiens par les Américains surtout, à l'île Royale. Le caractère désespérant de cette tâche, telle qu'il la décrivait, tenait à l'impossibilité pour les Indiens de changer leur mode de vie nomade, aussi longtemps que la fourrure allait être la source principale de leur revenu. Le Père Frémiot notait que la plupart des Indiens devaient leur gagne-pain à la Compagnie de la Baie d'Hudson qui en employait quelques-uns pour des engagements réguliers de trois ans, et en recrutait d'autres pour une courte période, le temps de faire le voyage d'aller-retour à Moose Factory. ${ }^{40}$ C'était surtout ce second genre de travail que le Père considérait comme spécialement dangereux et malsain. Il soulignait le caractère ardu du voyage, et supposait qu'il pouvait y avoir tout au moins un lien entre l'incidence tuberculeuse des jeunes Indiens et le travail qu'ils devaient faire. En guise de salaire, affirmaitil, on les payait en biens, les trompant par une marchandise, tantôt sans valeur, tantôt surévaluée. Selon son opinion, également, un certain nombre d'autres façons d'agir de la Compagnie de la Baie d'Hudson eurent un profond retentissement social (et finalement politique). Il prétendait que les élections des chefs de clans indiens subissaient les pressions de la Compagnie de la Baie d'Hudson, qui tantôt maintenait l'équilibre des forces, tantôt faisait pencher la balance, en choisissant et en récompensant les représentants les plus soumis. ${ }^{41}$

En face de ces messieurs [c'est à dire, les représentants du département des affaires indiennes] sur des chaises ou fauteuils sont assis nos deux chefs. Joseph, La Peau de Chat, est à la premiere ligne. Il est habillé comme les blancs, ainsi que tous les sauvages. C'est un homme d'environ 40 ans, grand et bien fait de sa personne, à la voix vibrante et sonore. La fougue eloquente, sa vehemente impétuosité l'ont fait choisir pour chef par les sauvages. Il ne lui manque qu'une âme plus fortement trempée dans la vie et les vertus chrétiennes. L'autre chef est un vieillard septuagénaire qu'on appelle l'Illinois. C'est tout simplement un de ces chefs de pelleteries établis par la Compagnie de la Baie d'Hudson. Il en reçoit tous les ans deux habits, dont l'un est rouge, galonné, et garni de boutons au metal. C'est-ce qui lui a fait donné le surnom de Miskouakkonaye (rouge habillé).

40 Ibid., II : 119, P. Frémiot à P. Micard, supérieur du Séminaire de St-Dié, le 2 février 1851 . 1849.

41 Ibid., I: 449, P. Frémiot au Supérieur à New-York, le 18 octobre 
Le Père Frémiot ne fut pas le seul Européen à observer et à décrire le ridicule costume de ces chefs; sir George Simpson et son épouse purent à peine retenir leur rire devant le ridicule de cette parure, ${ }^{42}$ mais ni l'un ni l'autre ne précisèrent quelle avait été la provenance de cette livrée. Le récit du Père Frémiot est le seul, dans l'état actuel de nos connaissances, qui fasse un lien explicite entre le costume et la collaboration avec la Compagnie, laissant entendre que ce vêtement rouge, insigne de servilité, était à la fois ridicule et humiliant pour les Indiens eux-mêmes.

Un autre commentaire du Père Frémiot, lors de la même conférence, nous montre son inquiétude sur l'orientation de la politique de la Compagnie de la Baie d'Hudson. Le commerçant en chef du fort avait dressé une liste des Indiens du lieu appelés à prendre part à cette conférence, tandis que tous les noms des Métis en étaient exclus. Le Père considère cela comme étant une décision de la Compagnie, et demande : "Est-ce politique ? Et craindrait-on que plus instruits que les sauvages, ils ne sussent mieux défendre les droits?" ${ }^{43}$ On ne trouve rien dans les archives de la Compagnie de la Baie d'Hudson à Thunder Bay, ou dans celles du département des affaires indiennes pour confirmer ou dénier la supposition du Père. Dans le journal de sir George Simpson, on note une référence à l'exclusion de certaines personnes (non Métis) d'une conférence, mais l'usage de la voix passive cache l'identité des protestataires, qu'ils soient Indiens ou représentants de la Compagnie. ${ }^{44}$ Le lecteur doit donc se demander si le Père Frémiot est digne de foi, et si sa position d'observateur rend son témoignage indiscutable.

Cette même conférence durant laquelle le Père avait été un témoin attentif, marquait le début d'une formulation de politique indigène pour Thunder Bay qui irait pour la première fois au-delà de l'emprise de la Compagnie de la Baie d'Hudson. A l'automne de 1849, le capitaine Thomas G. Anderson du département des affaires indiennes et deux associés poussèrent une pointe dans le nord-ouest pour négocier la reddition de territoires à la couronne. Le gouvernement canadien s'était rendu compte de la nécessité de telles ententes après la contestation des

42 Simpson, op. cit., I: 34; Grace L. Nute, (ed.) "Frances Simpson's 1830 Journal", in Beaver Magazine (mars 1954): 13-16.

43 A.S.J.C.F., Nouvelles Missions, I: 450, P. Frémiot au Supérieur à New York, le 18 octobre 1849.

44 Archives publiques du Canada, Microfilm of Hudson's Bay Company Records, D-3-2, Simpson Journal (May 28, 1841). La version publiée de ce journal ne comprenait pas cet item. 
concessions minières de Sault-Sainte-Marie par les Indiens. Le Père Frémiot affirmait qu'il ne savait rien de ces disputes. Il sembla même un peu surpris en lisant ces choses dans la Gazette de Montréal, ${ }^{45}$ car les mines qu'il connaissait étaient plutôt en train de fermer leur portes que de réclamer de nouvelles concessions. Quoi qu'il en soit, sur la question des ententes avec les Indiens, il était bien informé. Il avait des opinions bien arrêtées et peu favorables sur les traités américano-indiens. Il avait entendu parler des orgies qui se passaient chaque année à La Pointe lors de la distribution des redevances; ${ }^{46}$ il condamnera plus tard la politique américaine en termes non-équivoques. ${ }^{47}$ Cette conviction l'amena, du moins au début, à donner une approbation modérée aux plans canadiens qu'il considérait, au pire, comme préférables à ceux des Américains. Il fut rassuré de savoir que les redevances ne seraient pas distribuées en un seul point, mais en de nombreux endroits, par les bons soins des fonctionnaires de la Compagnie de la Baie d'Hudson..$^{48}$ Il était enclin à croire, avec le capitaine Anderson, que les paiements en biens plutôt qu'en monnaie étaient meilleurs, encore qu'il fût continuellement conscient des abus relatifs des deux systèmes. Il était convaincu dès le début que la somme que le gouvernement se proposait de verser était trop mince, bien qu'il vît quelques avantages à cette entente. ${ }^{49}$
Voila donc nos pauvres sauvages à la veille de recevoir, non une fortune toute faite qui les dispense de travailler ainsi que quelques-uns se l'imaginaient bonnement, mais quelques fai- bles secours qui, du moins, les aideront à s'habiller. Car, ici, la difficulté n'est pas de vivre, mais de se vêtir. La culture et la pêche fourniront une nourriture suffisante, mais le vêtement il en coute davantage de procurer, par la raison que la Compagnie de Baie d'Hudson a jusqu'ici le monopole des fourrures, et par conséquent du commerce.

Le jugement du Père Frémiot au sujet, et du traité et du Chef Peau de Chat qui avait donné son assentiment à ce traité,

45 Montreal Gazette, July 7, 1849.

46 A.S.J.C.F., Nouvelles Missions, I: 399-400, P. Choné à un père Jésuite, La Pointe, le 14 septembre 1848.

47 Ibid., II : 105-7, P. Frémiot à P. Micard, supérieur du Séminaire de St-Dié, le 2 février 1851.

48 Ibid., I: 452-3, P. Frémiot au Supérieur à New York, le 18 octobre 1849 ; P.A.C., Microfilm of Hudson's Bay Company Records, B-231-a-20, Fort William Post Journal (September 26, 1850).

49 A.S.J.C.F., Nouvelles Missions, I: 453, P. Frémiot au Supérieur à New York, le 18 octobre 1849. 
se fit plus dur avec le temps. Deux années plus tard, il écrivait: ${ }^{50}$

La mort providentielle de l'ancien chef, Joseph Peau de Chat, malgré l'indigence dont il a doté les sauvages par le honteux traité du Sault Ste. Marie, nous laisse jouir d'une paix dont les charmes sont d'autant mieux sentis qu'on y était moins habitués jusqu'alors.

Le ton amer de cette notice nécrologique contraste singulièrement avec le ton général de sa correspondance, encore que l'on ne puisse soupçonner les événements qui l'aient occasionné. Une insulte personnelle n'aurait pu produire pareil résultat. Le capitaine Anderson s'était assuré que le Jésuite qu'il nomme le Père Frimeault ne pourrait donner aucun conseil aux Indiens durant la conférence. "Finding he meddled with our business I could not forego the pleasure of informing him he had no business to interfere... but still he did not move his body, though his tongue was less busy." " ${ }^{1}$ La description que le Père fait de ces événements est empreinte d'aménité: "J'assistai aux séances comme simple spectateur, et j'acceptai l'offre de M. iviackenzie de partager le dîner de ces messieurs." 52 Au cours's de la longue lettre qu'il consacre aux événements de septembre 1849, jamais le Père Frémiot ne consent à ce que le ressentiment, provoqué par la brusquerie du capitaine Anderson, s'exprime en attaques contre les négociateurs de la conférence. Sans cesse, il dénonce les pressions que l'on fit, durant la conférence, contre Peau de Chat. C'était, selon lui, un faible de caractère qui mettait quelquefois les négociations en péril. Ce n'est donc pas la désillusion qui suit la destruction d'un idéal qui lui fit écrire la notice nécrologique plutôt amère de 1851 .

Le Père Frémiot a laissé un récit extraordinairement cornplet et poignant de la conférence des Indiens à Fort William: le commissaire du gouvernement et ses assistants étaient d'un côté de la pièce, les deux chefs indiens leur faisaient face, et "derrière les chefs, tout autour de la salle, les sauvages sont assis par terre, adossés contre le mur". ${ }^{53} \mathrm{Il}$ parut bientôt évident que le capitaine Anderson fit le meilleur usage du mécanisme que mettaient à sa disposition les précédents de la Compagnie de la Baie d'Hudson, s'efforçant de convaincre les Indiens 1851.

50 Ibid., II : 182, P. Frémiot à un père Jésuite en France, le 8 décembre 51 Toronto Public Library, Diary of Captain Thomas G. Anderson (September 24, 1849).

52 A.S.J.C.F., Nouvelles Missions, I: 448, P. Frémiot au Supérieur à New York, le 18 octobre 1849.

53 Ibid., 449. 
qu'Illinois était leur chef, plutôt que Peau de Chat. Peau de Chat exprima son opinion sur le questionnaire qu'Anderson lui avait soumis et lui fit part de ses réponses aux questions. ${ }^{54} \mathrm{Il}$ devint bientôt clair que ses demandes dépassaient de beaucoup ce que le gouvernement avait l'intention de donner. Le Père Frémiot cite les remarques du capitaine Anderson à la fin du premier jour de la session : ${ }^{55}$

Il est deux choses qui ne nous font pas plaisir et que verra pas non plus le bon œil, à ce que nous pensons, notre Père qui est à Montréal. La première c'est qu'il connait point, c'est qu'il n'a pas approuvé celui des deux chefs auxquels vous donnez la préférence. La seconde, c'est que vous demandez un trop haut prix pour vos terres.

Peau de Chat, naturellement, s'irrita contre cette affirmation et le matin suivant, après la messe, avant que la conférence ne recommence, il vint parler au Père Frémiot de ce qu'il voulait faire. Il interprétait l'affirmation d'Anderson comme une peur de l'influence qu'il pouvait avoir et conclut que la chose la plus sage à faire serait de retirer sa candidature de la liste des chefs. Le Père vit bien que ce geste allait renforcer la position d'Anderson plutôt que de l'affaiblir. Il supplia Peau de Chat de rester à son poste, au moins jusqu'à ce que les négociations fussent terminées, soulignant qu'il était naturel qu'un Anglais préfère un chef ayant quelques liens avec le protestantisme, mais que ce désir n'aurait aucune portée pratique aussi longtemps que les Indiens continueraient à vouloir Peau de Chat comme leur principal porte-parole. Le conseil fut suivi, puisqu'à la réunion suivante, il ne fut plus question de résignation. Au contraire, on a pu rapporter ces paroles du chef: "Ni le Grand Chef qui est à Montréal ni la Reine prétendent rien changer les élections des sauvages, ni influencer en rien leurs délibérations."

Les événements de septembre 1849 furent les plus mémorables que le Père Frémiot ait connus à Thunder Bay, et le récit qu'il en fait est le document le plus important de sa correspondance. Ses impressions premières furent de toute évidence réexaminées durant les deux années suivantes, mais malheureusement il n'a laissé aucune explication de ces changements d'opinions. Parmi les territoires qu'il a parcourus, en ces années-là, rien ne lui a permis de croire à l'imminence d'un défi au mono-

54 A.S.J.C.F., A-16-1, Traduction française du rapport de Peau de Chat, sur les questions que lui a posées l'agent des affaires indiennes, lors de sa visite de 1849 .

55 A.S.J.C.F., Nouvelles Missions, I: 450, P. Frémiot au Supérieur à New York, le 18 octobre 1849. 
pole de la Compagnie de la Baie d'Hudson. Aucune entreprise commerciale autre que le commerce des fourrures ne lui semblait possible; aucune arrivée massive d'immigrants ne semblait imminente. Il continua de desservir les petites colonies telles que celle du lac Nipigon, constituée d'un commis en charge et de six engagés: deux Ecossais protestants et quatre Catholiques romains, dont un Canadien français, un Métis et deux Indiens. ${ }^{56}$ Il ne faisait pas de distinction entre l'attitude des représentants du gouvernement canadien et celle des membres de la Compagnie. Il avait ses préjugés, ils avaient les leurs, chacun restait sur ses positions. La situation dramatique du conseil de Fort William et la profonde satisfaction qu'il retirait de son travail missionnaire ne l'empêchèrent pas, à certains moments, de se convaincre que bien peu pouvait être accompli et de ressentir une solitude extrême. Un tel découragement ne s'exprimait pas directement, mais cela ressort d'une lettre dans laquelle le Père Frémiot compare sa destinée à celle d'un ancien compagnon d'études, alors professeur à Brugelette. ${ }^{57}$ Cela perce dans la description de deux mineurs alsaciens isolés qui s'entretinrent avec lui pendant des heures. "C'est à part nos Pères les seuls français que j'ai vus depuis que je suis parmi les sauvages." 58

En juin 1852, le Père Frémiot reçut l'ordre de se diriger vers le Sault-Sainte-Marie, et il partit aussitôt les arrangements faits. Sur l'île Royale, attendant d'être transporté, il jeta un regard en arrière sur ses années à Thunder Bay, notant même la générosité du Père Choné avec lequel, désormais, il n'allait plus être associé. ${ }^{59}$ De l'île Manitouline, il racontait qu'il avait fait ses adieux à tous les amis de Fort William et de Nipigon. ${ }^{60}$ De là, il se mit en route pour son dernier voyage missionnaire durant lequel il se noya en 1854. Dans les comptes rendus des missions de Fort William et de Nipigon, on ne trouve presque rien qui ait trait au Père Frémiot. Parmi ceux qui ont collaboré aux débuts de la mission des Jésuites, on ne signale aucun nom. ${ }^{61}$ Quand les pionniers vinrent s'établir en permanence vers la fin 1852.

56 Ibid., II : 203, P. Frémiot au Supérieur en Amérique, le 19 mars 1851. 1852.

57 Ibid., II : 148, P. Frémiot à un Jésuite scolastique, le 18 octobre 58 Ibid., 156-7.

59 Ibid., II : 214, P. Frémiot au Supérieur en Amérique, le 2 juillet

60 Ibid., II: 229, P. Frémiot à un Scolastique à Laval, le 7 août 1852.

61 Canada, Parliament, Appendix to Journals (1858), XVI, $\mathrm{n}^{\circ} 6$, Appendice 28, Réponses du Père Choné aux questions du Département des Indiens, 6 juin 1857. 
des années 1850 , ce fut le Père Choné qu'ils rencontrèrent et admirèrent. Le travail de l'infatigable Père Du Ranquet, durant les vingt années qui suivirent, prit les dimensions d'une légende à Thunder Bay, tandis que celui de son prédécesseur fut, de fait, oublié. Pourtant, des lettres que le Père Frémiot écrivit quand lui et la mission étaient jeunes, il ressort des peintures vivantes du pays lui-même, de la société de l'époque, des Ojibways, de la Compagnie de la Baie d'Hudson, et du démarrage de nouveaux projets, tant jésuites que canadiens. Il lui était impossible de mesurer à sa juste valeur l'impact du nouveau sur l'ancien, mais il notait ce qu'il voyait avec une remarquable clarté de vision, une grande douceur de tempérament, un délicat maniement de l'ironie, une humeur aux variations subtiles. 